\title{
On the Omori-Yau Maximum Principle in Riemannian submersions
}

\section{G. Pacelli Bessa and Paolo Piccione}

Departamento de Matemáticas,

Universidad de Murcia, Campus de Espinardo

30100 Espinardo, Murcia,

Spain

\begin{abstract}
We discuss some sufficient conditions for the validity of the weak and the strong Omori-Yau maximum principle in Riemannian submersions.
\end{abstract}

\section{Introduction}

Roughly speaking, a Riemannian manifold is said to satisfy the Omori-Yau maximum principle if every smooth real-valued function on the manifold which is bounded from above admits a maximum at infinity. See Section 3. for the precise definition; a complete reference is the book by Pigola, Rigoli and Setti, see [9]. The principle has proved to be a powerful tool in Submanifold Theory, especially for proving curvature estimates of submanifolds satisfying suitable boundedness assumptions, see for instance $[1,2,5]$.

Clearly, the validity of the principle has to do with the geometry at infinity of the manifolds, i.e., it is not affected by arbitrary perturbations of the metric inside a compact set and it is a very intriguing question to determine precisely which geometrical properties guarantee the validity of the principle, in one of its several and non equivalent formulations. Moreover, the validity of the principle does not behave well with respect to some natural operations in Riemannian geometry, like for instance passing to submanifolds, or taking products.

Some sufficient conditions for the validity of the principle are known; they are discussed in detail in [9]. One condition has to do with some decay control of the curvature at infinity. A second condition requires the existence of a proper smooth real-valued function on the manifold $M$ with control on the growth of the gradient and of the second derivative at infinity, see Theorem 3.2. The condition

Received by the editors February 21st, 2010. 
is stated more precisely in terms of a pair of functions $\gamma: M \rightarrow(0,+\infty)$ and $G:(0,+\infty) \rightarrow(0,+\infty)$, which is called an OY-pair in the present paper.

In this paper we will discuss a generalization of this condition, as follows. In many applications, the proper function $\gamma$ with bounded growth is obtained using the distance function from a point, or from a compact subset, of the Riemannian manifold. Properness in this case is equivalent to the completeness of the metric, and the smoothness property holds outside the cut locus.

The gradient of the distance function has constant length equal to 1 . The key observation here is that any function $f: M \rightarrow \mathbb{R}$ whose gradient is a vector field of constant norm equal to 1 is a Riemannian submersion, having total space $M$ and base (an interval of) $\mathbb{R}$, endowed with the Euclidean metric. The level surfaces of the distance function, that are the fibers of the Riemannian submersion, are the geodesic spheres. The Hessian of the distance function is the second fundamental form of these spheres, and the Laplacian is the mean curvature.

This gives the following geometrical interpretation: the Omori-Yau principle holds in Riemannian manifolds with controlled growth at infinity of the second fundamental form (or the mean curvature) of the geodesic spheres.

A natural question is whether a similar criterion holds for arbitrary Riemannian submersions. In the present paper we are going to discuss this question, giving an affirmative answer. Our main result is that, given a Riemannian submersion $\pi: M \rightarrow N$ having compact fibers with controlled second fundamental form or mean curvature, then the Omori-Yau principle holds in $M$ provided that $N$ admits an OY-pair.

In Riemannian submersions, the second fundamental form of the fibers is given by one of the two fundamental tensors of the submersions, usually denoted by $T$, see [7]. For the strong version of the Omori-Yau principle, a certain control on the growth of the other fundamental tensor of the Riemannian submersion, denoted by $A$, which is the integrability tensor of the horizontal distribution is also required.

\section{Riemannian submersions}

Given smooth manifolds $M$ and $N$, a smooth surjective map $\pi: M \rightarrow N$ is a submersion if the differential $\mathrm{d} \pi(q)$ has maximal rank for every $q \in M$. If $\pi: M \rightarrow N$ is a submersion, then for all $p \in N$ the inverse image $\mathcal{F}_{p}=\pi^{-1}(p)$ is a smooth embedded submanifold of $M$, that will be called the fiber at $p$. If $M$ and $N$ are Riemannian manifolds, then a submersion $\pi: M \rightarrow N$ is called a Riemannian submersion if for all $p \in N$ and all $q \in \mathcal{F}_{p}$, the restriction of $\mathrm{d} \pi(q)$ to the orthogonal subspace $T_{q} \mathcal{F}_{p}^{\perp}$ is an isometry onto $T_{p} N$.

Given $p \in N$ and $q \in \mathcal{F}_{p}$, a tangent vector $\xi \in T_{q} M$ is said to be vertical if it is tangent to $\mathcal{F}_{p}$, and it is horizontal if it belongs to the orthogonal space $\left(T_{q} \mathcal{F}_{p}\right)^{\perp}$. 
Let $\mathcal{D}=(T \mathcal{F})^{\perp} \subset T M$ denote the smooth distribution on $M$ consisting of horizontal vectors. The orthogonal distribution $\mathcal{D}^{\perp}$ is clearly integrable, the fibers of the submersion being its maximal integral leaves. Given $\xi \in T M$, its horizontal and vertical components are denoted respectively by $\xi^{h}$ and $\xi^{v}$. The second fundamental form of the fibers is a symmetric tensor $\mathcal{S}^{\mathcal{F}}: \mathcal{D}^{\perp} \times \mathcal{D}^{\perp} \rightarrow \mathcal{D}$, defined by

$$
\mathcal{S}^{\mathcal{F}}(v, w)=\left(\nabla_{v}^{M} W\right)^{h},
$$

where $W$ is a vertical extension of $w$ and $\nabla^{M}$ is the Levi-Civita connection of $M$.

For any given vector field $X \in \mathfrak{X}(N)$, there exists a unique horizontal vector field $\widetilde{X} \in \mathfrak{X}(M)$ which is $\pi$-related to $X$, this is, for any $p \in N$ and $q \in \mathcal{F}_{p}$, then $\mathrm{d} \pi_{q}\left(\widetilde{X}_{q}\right)=X_{p}$, called horizontal lifting of $X$. A horizontal vector field $\widetilde{X} \in \mathfrak{X}(M)$ is called basic if it is $\pi$-related to some vector field $X \in \mathfrak{X}(N)$.

Let us now consider the geometry of the fibers. First, we observe that the fibers are totally geodesic submanifolds of $M$ exactly when $\mathcal{S}^{\mathcal{F}}=0$. The mean curvature vector of the fiber is the horizontal vector field $H$ defined by

$$
H(q)=\sum_{i=1}^{k} \mathcal{S}^{\mathcal{F}}(q)\left(e_{i}, e_{i}\right)=\sum_{i=1}^{k}\left(\nabla_{e_{i}}^{M} e_{i}\right)^{h}
$$

where $\left(e_{i}\right)_{i=1}^{k}$ is a local orthonormal frame for the fiber through $q$. Observe that $H$ is not basic in general. For instance, when $n=1$, i.e., when the fibers are hypersurfaces of $M$, then $H$ is basic if and only if all the fibers have constant mean curvature. The fibers are minimal submanifolds of $M$ when $H \equiv 0$.

Given a smooth function $f: N \rightarrow \mathbb{R}$, denote by $\tilde{f}=f \circ \pi: M \rightarrow \mathbb{R}$ its lifting to $M$. It is easy to see that the gradient $\operatorname{grad}^{M} \tilde{f}$ of $\tilde{f}$ is the horizontal lifting of the gradient $\operatorname{grad}^{N} f$. If we denote with a tilde $\tilde{X}$ the horizontal lifting of a vector field $X \in \mathfrak{X}(N)$, then the previous statement can be written as

$$
\operatorname{grad}^{M} \tilde{f}=\widetilde{\operatorname{grad}^{N}} f .
$$

The Hessian of $\tilde{f}$ can be computed explicitly as follows. If $X \in T M$ is a horizontal vector, then:

$$
\operatorname{Hess}^{M} \tilde{f}(X, X)=\operatorname{Hess}^{N} f(\mathrm{~d} \pi(X), \mathrm{d} \pi(X)),
$$

while the Hessian formula for $V$ vertical involves the second fundamental form of the fibers:

$$
\operatorname{Hess}^{M} \tilde{f}(V, V)=-g^{M}\left(\operatorname{grad}^{M} \tilde{f}, \mathcal{S}^{\mathcal{F}}(V, V)\right) .
$$

The Laplacian of $\tilde{f}$, the trace of the Hessian of $\tilde{f}$, can now be computed using an orthonormal basis of $T M$ which consists of an orthonormal basis of the horizontal 
space and an orthonormal basis of the fiber. Using (2.3) and (2.4), we obtain the following relation between the Laplacian of $f$ and the Laplacian of $\tilde{f}$, see [3]

$$
\begin{aligned}
\left(\Delta^{M} \tilde{f}\right)_{q} & =\left(\Delta^{N} f\right)_{p}-g^{M}\left(\left(\operatorname{grad}^{M} \tilde{f}\right)_{q}, H_{q}\right) \\
& =\left(\Delta^{N} f\right)_{p}-g^{N}\left(\left(\operatorname{grad}^{N} f\right)_{p}, \mathrm{~d} \pi_{q}\left(H_{q}\right)\right) .
\end{aligned}
$$

It will be useful to have a formula for $\operatorname{Hess}^{M} \tilde{f}(X, V)$, with $X$ horizontal and $V$ vertical. To this aim, we need to recall the definition of the fundamental tensor $A$ of a Riemannian submersion. This is a $(1,2)$-tensor on $M$, defined by:

$$
A_{\xi} \eta=\left(\nabla_{\xi^{h}}^{M}\left(\eta^{h}\right)\right)^{v}+\left(\nabla_{\xi^{h}}^{M}\left(\eta^{v}\right)\right)^{h},
$$

for $\xi, \eta \in T M$. The tensor $A$ has the following properties (see [7]):

- $A_{\xi}$ is $g^{M}$-antisymmetric, and it reverses the horizontal and the vertical subspaces;

- $A_{\xi}=A_{\xi h}$

- given horizontal vector fields $X$ and $Y$, then $A_{X} Y=\frac{1}{2}[X, Y]^{v}$, where $[X, Y]$ is the Lie bracket. In particular, $A_{X} Y=-A_{Y} X$.

Last property says that, restricted to horizontal vectors, $A$ is the integrability tensor of the horizontal distribution. Using the above properties one sees easily that $A$ vanishes identically (i.e., $A_{\xi} \eta=0$ for all vectors $\xi, \eta \in T M$ ) if and only if the horizontal distribution is integrable.

When $Y$ is a basic vector field, then for any vertical vector $V$ one has:

$$
\left(\nabla_{V}^{M} Y\right)^{h}=\left(\nabla_{Y}^{M} V\right)^{h}=A_{Y} V .
$$

If we apply (2.7) to $Y=\operatorname{grad}^{M} \tilde{f}$ and use the properties of $A$, then for $X$ horizontal and $V$ vertical we obtain:

$$
\begin{aligned}
& \operatorname{Hess}^{M} \tilde{f}(X, V)=\operatorname{Hess}^{M} \tilde{f}(V, X)=g^{M}\left(A_{\operatorname{grad}^{M}} \tilde{f} V, Y\right) \\
& =-g^{M}\left(A_{\operatorname{grad}^{M}} \tilde{f} Y, V\right)=g^{M}\left(A_{Y}\left(\operatorname{grad}^{M} \tilde{f}\right), V\right)=-g^{M}\left(A_{Y} V, \operatorname{grad}^{M} \tilde{f}\right) .
\end{aligned}
$$

Note that, when the horizontal distribution is integrable, then $\operatorname{Hess}^{M} \tilde{f}(X, V)=$ 0 .

\section{The Omori-Yau maximum principle}

Definition 3.1. We say that the Omori-Yau Maximum Principle for the Hessian holds in a Riemannian manifold $\left(M, g^{M}\right)$ if given a smooth function $f: M \rightarrow \mathbb{R}$ with $\sup f<+\infty$ there exists a sequence $\left(p_{n}\right)_{n \in \mathbb{N}}$ in $M$ such that:

$$
M
$$


(a) $\lim _{n \rightarrow \infty} f\left(p_{n}\right)=\sup _{M} f$;

(b) $\left\|\operatorname{grad}^{M} f\left(p_{n}\right)\right\| \leq \frac{1}{n}$;

(c) $\operatorname{Hess}^{M} f\left(p_{n}\right)(e, e) \leq \frac{1}{n} g^{M}(e, e)$ for all $e \in T_{p_{n}} M$.

for all $n$. Similarly, the Omori-Yau principle for the Laplacian holds in $\left(M, g^{M}\right)$ if if the above properties hold, with (c) replaced by the weaker condition:

(c') $\Delta^{M} f\left(p_{n}\right) \leq \frac{1}{n}$.

A weaker form of the principle is obtained by dropping condition (b) from the requirements on the sequence $p_{n}$. We say that the weak Omori-Yau Principle for the Hessian (resp., for the Laplacian) in $\left(M, g^{M}\right)$ if for every smooth function $f: M \rightarrow \mathbb{R}$ with $\sup _{M} f<+\infty$ there exists a sequence $\left(p_{n}\right)_{n \in \mathbb{N}}$ in $M$ satisfying (a) and (c) (resp., (a) and (c')) above.

Let us recall that a Riemannian manifold $\left(M, g^{M}\right)$ is stochastically complete if, denoting by $p: M \times M \times(0,+\infty) \rightarrow \mathbb{R}$ the heat kernel associated to the Laplacian $\Delta^{M}$, then for every $(x, t) \in M \times(0,+\infty)$ :

$$
\int_{M} p(x, y, t) \mathrm{d} \mu(y)=1 \text {. }
$$

An important and beautiful result due to Pigola, Rigoli and Setti, see [8], states that stochastic completeness is equivalent to the weak Omori-Yau principle for the Laplacian, see also [9, Chapter 3] for related questions.

Some sufficient conditions are known for the Omori-Yau Maximum Principle to hold in a Riemannian manifold. We will use the following:

Theorem 3.2 (Pigola-Rigoli-Setti [9]). Assume that there exist smooth functions $G:(0,+\infty) \rightarrow(0,+\infty)$ and $\gamma: M \rightarrow(0,+\infty)$ such that:

(1) $G(0)>0, G^{\prime}(t) \geq 0$ for all $t \geq 0$;

(2) $\limsup _{t \rightarrow+\infty} \frac{t \cdot G(\sqrt{t})}{G(t)}<+\infty$;

(3) $\int_{0}^{+\infty} \frac{\mathrm{d} t}{\sqrt{G(t)}}=+\infty$;

(4) $\gamma$ is proper;

(5) $\left\|\operatorname{grad}^{M} \gamma\right\| \leq c \cdot \sqrt{\gamma}$ for some $c>0$ outside a compact subset of $M$;

(6) Hess $^{M} \gamma \leq c^{\prime} \cdot \sqrt{\gamma \cdot G(\sqrt{\gamma})}$ for some $c^{\prime}>0$ outside a compact subset of $M$. 
Then, the Omori-Yau Maximum Principle for the Hessian holds in $\left(M, g^{M}\right)$. A totally analogous statement holds in the case of the Omori-Yau principle for the Laplacian, with assumption (6) replaced by:

(6') $\Delta^{M} \gamma \leq c^{\prime} \cdot \sqrt{\gamma \cdot G(\sqrt{\gamma})}$ for some $c^{\prime}>0$ outside a compact subset of $M$.

Proof. See [9].

Note that any function $G$ satisfying (1) and (2) is unbounded:

$$
\lim _{t \rightarrow+\infty} G(t)=+\infty \text {. }
$$

For the sake of brevity, let us say that a pair of functions $(G, \gamma)$ satisfying (1)—(6) of Proposition 3.2 will be called an OY-pair for the Hessian in $\left(M, g^{M}\right)$. Similarly, a pair $(G, \gamma)$ satisfying (1)-(5) and (6') is called an OY-pair for the Laplacian in $\left(M, g^{M}\right)$.

Let us also recall from [9] the following result that gives a sufficient condition for the validity of the weak Omori-Yau principle for the Laplacian.

Theorem 3.3. (Khas'minskii, [6], Varopoulos, [12]) Let $\left(M, g^{M}\right)$ be a Riemannian manifold that admits a proper $C^{2}$-function $\gamma: M \rightarrow(0,+\infty)$ such that, outside some compact subset, $\Delta^{M} \gamma \leq \lambda \gamma$ for some positive constant $\lambda$. Then the weak Omori-Yau principle for the Laplacian holds in $\left(M, g^{M}\right)$.

Proof. See [9, Proposition 3.2].

\section{The Omori-Yau principle in Riemannian submersions}

Proposition 4.1. Let $\pi: M \rightarrow N$ be a Riemannian submersion with compact fibers, and define $\mathfrak{h}: N \rightarrow \mathbb{R}$ by

$$
\mathfrak{h}(p)=\max _{q \in \mathcal{F}_{p}}\left\|H_{q}\right\|_{M}
$$

Let $(G, \Gamma)$ be an OY-pair for the Laplacian in $\left(N, g^{N}\right)$, and assume that $\mathfrak{h}$ satisfies:

$$
\mathfrak{h} \leq d \cdot \sqrt{G(\sqrt{\Gamma})}
$$

outside a compact subset of $N$, for some positive constant $d$. Then, $(G, \Gamma \circ \pi)$ is an OY-pair for the Laplacian in $\left(M, g^{M}\right)$.

Proof. The function $\gamma=\Gamma \circ \pi: M \rightarrow(0,+\infty)$ is proper; namely, given a compact subset $K \subset(0,+\infty)$, the inverse image $\gamma^{-1}(K)$ is given by $\pi^{-1}\left(\Gamma^{-1}(K)\right)$. Since $\Gamma$ is proper, $K^{\prime}=\Gamma^{-1}(K)$ is compact, and $\pi^{-1}\left(K^{\prime}\right)$ is compact because $\pi$ has compact fibers. 
By (2.2), $\left\|\operatorname{grad}^{M} \Gamma\right\|_{M}=\left\|\operatorname{grad}^{N} \gamma\right\|_{M} \leq c \cdot \sqrt{\gamma}=c \cdot \sqrt{\Gamma}$ for some $c>0$ outside a compact subset of $M$. Here we use again the compactness of the fibers.

Cauchy-Schwarz inequality and assumption (4.2) give:

$$
\left|g^{N}\left(\operatorname{grad}^{N} \Gamma, \mathrm{d} \pi(H)\right)\right| \leq \alpha \cdot \sqrt{\Gamma \cdot G(\sqrt{\Gamma})},
$$

for some $\alpha>0$, outside a compact subset of $N$. Finally, using (2.5) and (4.3), we get

$$
\Delta^{M} \gamma \leq c^{\prime} \sqrt{\gamma \cdot G(\sqrt{\gamma})}
$$

outside a compact subset of $M$. Hence, $(G, \gamma)$ is an OY-pair for the Laplacian in $\left(M, g^{M}\right)$.

Using Proposition 3.3, we can prove an analogue to Proposition 4.1 for the weak Omori-Yau principle.

Proposition 4.2. Let $\pi: M \rightarrow N$ be a Riemannian submersion with compact fibers, and assume that $\Gamma: N \rightarrow(0,+\infty)$ is a proper $C^{2}$-function satisfying $\Delta^{N} \Gamma \leq \lambda \Gamma$ outside some compact subset of $N$ for some positive $\lambda$. If (4.2) holds outside a compact subset of $N$, then the weak Omori-Yau principle for the Laplacian holds in $\left(M, g^{M}\right)$.

Proof. Arguing as in the proof of Proposition 4.1, one proves that $\gamma=\Gamma \circ \pi$ is proper, and $\Delta^{M} \gamma \leq \lambda^{\prime} \gamma$ outside some compact subset of $M$, for some $\lambda^{\prime}>0$. The conclusion follows from Proposition 3.3.

An analogue of Propositions 4.1 and 4.2 concerning the Omori-Yau principle for the Hessian is obtained by replacing assumption (4.2) with suitable boundedness assumptions on the norm of the second fundamental form of the fibers and of the tensor $A$. To this aim, let us introduce the following functions $\mathfrak{s}, \mathfrak{a}: N \rightarrow \mathbb{R}$. Set:

$$
\mathfrak{s}(p)=\max _{q \in \mathcal{F}_{p}} \max \left\{\left\|\mathcal{S}^{\mathcal{F}}(V, V)\right\|_{M}: V \in T_{q} M,\|V\|_{M}=1,\right\} .
$$

and

$$
\mathfrak{a}(p)=\max _{q \in \mathcal{F}_{p}} \max \left\{\left\|A_{X} V\right\|_{M}: X, V \in T_{q} M,\|X\|_{M}=\|V\|_{M}=1,\right\} .
$$

Proposition 4.3. Let $\pi: M \rightarrow N$ be a Riemannian submersion with compact fibers. Let $(G, \Gamma)$ be an OY-pair for the Hessian in $\left(N, g^{N}\right)$, and assume that $\mathfrak{s}$ and $\mathfrak{a}$ satisfy:

$$
\mathfrak{s} \leq d \cdot \sqrt{G(\sqrt{\Gamma})}, \quad \mathfrak{a} \leq d \cdot \sqrt{G(\sqrt{\Gamma})},
$$

outside a compact subset of $N$, for some positive constant $d$. Then, $(G, \Gamma \circ \pi)$ is an OY-pair for the Hessian in $\left(M, g^{M}\right)$. 
Similarly, if $\Gamma: N \rightarrow(0,+\infty)$ is a proper $C^{2}$-function satisfying $\operatorname{Hess}^{N} \Gamma \leq$ $\lambda \Gamma$ outside some compact subset of $N$ for some positive $\lambda$, and if inequalities (4.6) hold outside a compact subset of $N$, then the weak Omori-Yau principle for the Hessian holds in $\left(M, g^{M}\right)$.

Proof. The proof is totally analogous to that of Propositions 4.1 and 4.2, using formulas (2.3), (2.3) and (2.8). Now the trace is not taken in the Hessian formulas, and the mixed term (2.8) has to be taken into account.

Corollary 4.4. Assume that $(M, g)$ possesses an OY-pair (either for the Hessian or the Laplacian). Then any finite covering of $M$ does, as well as the unit vector bundle $T_{1} M$ endowed with the Sasaki metric.

Proof. Any finite covering $\pi: \widetilde{M} \rightarrow M$, endowed with the pull-back metric $\pi^{*}(g)$, can be seen as a Riemannian submersion with compact totally geodesic fibers. Similarly, The Sasaki metric of $T_{1} M$ makes the canonical projection $\pi$ : $T_{1} M \rightarrow M$ a Riemannian submersion with totally geodesic fibers.

Fiberwise deformations of Riemannian submersions. Given a Riemannian submersion $\pi: M \rightarrow N$, some interesting deformations of the metric of the total space have been studied in the literature. For instance, the so-called canonical variation of the metric $g^{M}$ by a 1-parameter family of metrics $\left(g_{t}^{M}\right)_{t>0}$ has been introduced in order to study the existence of Einstein metrics, see [4]. Let us define a more general class of deformations as follows.

Given a smooth positive function $\psi: N \rightarrow \mathbb{R}^{+}$on the base of a Riemannian submersion $\pi: M \rightarrow N$, let $g_{\psi}^{M}$ denote the metric tensor in $M$ defined by the following relations:

$$
g_{\psi}^{M}(V, \xi)=\psi \cdot g^{M}(V, \xi), \quad g_{\psi}^{M}(X, \xi)=g^{M}(X, \xi),
$$

for all $\xi, V, X \in T M$, with $X$ horizontal and $V$ vertical. It is easy to see that $g_{\psi}^{M}$ defines a metric on $M$ for which $\pi: M \rightarrow N$ is again a Riemannian submersion, with the same horizontal distribution. Moreover, $g^{M}$ and $g_{\psi}^{M}$ coincide on the horizontal distribution, so that in fact the deformation only occurs on the fibers. Observe that in the particular case that $\psi$ is a constant function, then $g_{\psi}^{M}$ coincides with one of the metrics of the canonical deformation.

As to the fundamental tensors of $\pi: M \rightarrow N$ corresponding to the deformed metric $g_{\psi}^{M}$, clearly the integrability tensor $A$ is unchanged. The second fundamental form of the fibers are easily computed:

Lemma 4.5. Let $\mathcal{S}_{\psi}^{\mathcal{F}}$ denote the second fundamental form of the fibers of $\pi$ relatively to the metric $g_{\psi}^{M}$ and let $H_{\psi}$ denote its trace, i.e., the mean curvature of the 
fiber with respect to $g_{\psi}^{M}$. The following relations hold:

$$
\begin{aligned}
& \mathcal{S}_{\psi}^{\mathcal{F}}\left(v_{1}, v_{2}\right)=\psi \cdot \mathcal{S}^{\mathcal{F}}\left(v_{1}, v_{2}\right)-\frac{1}{2} g^{M}\left(v_{1}, v_{2}\right) \cdot \operatorname{grad}^{N} \psi, \\
& H_{\psi}=\psi \cdot H-\frac{1}{2} n_{\mathcal{F}} \cdot \operatorname{grad}^{N} \psi,
\end{aligned}
$$

for vertical vectors $v_{1}$ and $v_{2}$, where $n_{\mathcal{F}}$ is the dimension of the fibers, i.e., $n_{\mathcal{F}}=$ $\operatorname{dim}(M)-\operatorname{dim}(N)$.

Proof. Denote by $\nabla$ and $\nabla^{\psi}$ the Levi-Civita connection of $g^{M}$ and of $g_{\psi}^{M}$ respectively. An immediate computation using Koszul formula gives:

$$
2 g_{\psi}^{M}\left(\left(\nabla^{\psi}\right)_{v_{1}} V_{2}, X\right)=2 \psi g^{M}\left(\nabla_{v_{1}} V_{2}, X\right)-X(\psi) g^{M}\left(v_{1}, V_{2}\right),
$$

for all $v_{1}$ vertical and $X$ horizontal vectors, and for all $V_{2}$ vertical vector field. Formulas (4.7) follow readily.

Interesting deformations can be applied to Riemannian submersions having totally geodesic fibers, i.e., with vanishing second fundamental form. This is the case of two important non compact examples of Riemannian submersions, namely, the three-dimensional Heisenberg group $\mathrm{Heis}^{3}$, which is the total space of a Riemannian submersion with base the Euclidean $\mathbb{R}^{2}$, and the $2 \times 2$ special linear group $\mathrm{SL}(2, R)$. There exists a 2-parameter family of left-invariant Riemannian metrics $g_{\kappa, \tau}$ on $\mathrm{SL}(2, R)$, with $\kappa<0$ and $\tau \neq 0$, whose isometry group has dimension 4. Endowed with these metrics, $\operatorname{SL}(2, \mathbb{R})$ is one of the eight homogeneous Riemannian 3-geometries, as classified in [10]. An explicit description of these metrics can be found, for instance, in [11]. Endowed with the metrics $g_{\kappa, \tau}$ is isometric to the total space of a Riemannian fibration $\pi: \operatorname{SL}(2, \mathbb{R}) \rightarrow \mathbb{H}^{2}(\kappa)$ with basis the two-dimensional spaceform of constant curvature $\kappa<0$, and with geodesic fibers diffeomorphic to the circle $\mathbb{S}^{1}$. Let $\rho: \mathbb{H}^{2}(\kappa) \rightarrow \mathbb{R}$ be the distance to a fixed point $x_{0} \in \mathbb{H}^{2}(\kappa)$ and let $\psi: \mathbb{H}^{2}(\kappa) \rightarrow \mathbb{R}^{+}$be a smooth function whose gradient satisfies

$$
\|\operatorname{grad} \psi\| \leq c \cdot \sqrt{G(\sqrt{\rho})}
$$

off a compact set, where $G$ is a function satisfying the conditions (1), (2), (3) in Theorem 3.2.

Corollary 4.6. For all $\tau \neq 0$, denote by $g_{\kappa, \tau, \psi}$ the metric on $\operatorname{SL}(2, \mathbb{R})$ obtained by fiberwise deformation of $g_{\kappa, \tau}$ via $\psi$. Then, the Omori-Yau maximum principle for the Laplacian holds in $\left(\mathrm{SL}(2, \mathbb{R}), g_{\kappa, \tau, \psi}\right)$. In particular, $\left(\mathrm{SL}(2, \mathbb{R}), g_{\kappa, \tau, \psi}\right)$ is stochastically complete.

Proof. Use Proposition 4.1, formulas (4.7) and the $(G, \rho)$ as OY-pair for the Hyperbolic plane. 


\section{References}

[1] L. J. AlíAs, G. P. Bessa, M. DAJCzer, The mean curvature of cylindrically bounded submanifolds, Math. Ann. 2009.

[2] L. J. Alías, G. P. Bessa, J. F. Montenegro, An estimate for the sectional curvature of cylindrically bounded submanifolds, preprint 2009.

[3] G. Pacelli Bessa, J. Fabio Montenegro, Paolo Piccione, Riemannian submersions with discrete spectrum, preprint 2010, arXiv: $1001.0853 \mathrm{v} 1$.

[4] A. L. BESSE, Einstein manifolds, Ergebnisse der Mathematik und ihrer Grenzgebiete (3), 10. Springer-Verlag, Berlin, 1987.

[5] L. JoRge, D. Koutrofiotis, An estimate for the curvature of bounded submanifolds, Amer. J. Math. 103 (1980) 711-725.

[6] R. Z. KHAS'MINSKII, Ergotic properties of recurrent diffusion and stabilization of solutions to the Cauchy problem for parabolic equations, Theor. Prob. Appl. 5, (1960), 179195.

[7] B. O'NeILL, The fundamental equations of a submersion, Michigan Math. J. 13 (1966), 459-469.

[8] S. Pigola, M. Rigoli, A. Setti, A remark on the maximum principle and stochastic completeness, Proc. Amer. Math. Soc. 131 (2003), no. 4, 1283-1288.

[9] S. Pigola, M. Rigoli, A. Setti, Maximum Principle on Riemannian Manifolds and Applications, Memoirs Amer. Math. Soc. 822 (2005).

[10] P. SCOTT, The geometries of 3-manifolds, Bull. London Math. Soc. 15 (5) (1983), 401487.

[11] F. TORRALBO, Rotationally invariant constant mean curvature surfaces in homogeneus 3-manifolds, preprint 2009, arXiv:0911.5128v1.

[12] N. TH. VAROPOUlos, Potential theory and diffusion on Riemannian manifolds, Conference on Harmonic Analysis in honor of Antoni Zygmund. vol. I, II, Wadsworth Math. Ser., Wadsworth, Belmont, CA, (1983), 821-837. 